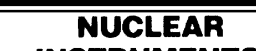

\title{
LHCb Vertex Locator prototyping, testing and production
}

\author{
Doris Eckstein* \\ CERN, PH/LBD, 1211 Geneva 23, Switzerland \\ On behalf of the LHCb VELO group \\ Available online 28 December 2005
}

\begin{abstract}
$\mathrm{LHCb}$ is the experiment at the Large Hadron Collider (LHC) dedicated to the study of CP violation in the B-system. Its silicon vertex detector, named VELO for VErtex LOcator, will precisely reconstruct the primary pp interaction vertex, the displaced B-decay vertex and tracks originating from them. The use of the VELO in the LHCb trigger together with its operation in a harsh radiation environment puts additional constraints on the design. The project is soon entering the production phase. Recent results on the tests of prototype modules and components are presented with a focus on the sensor and front-end chip performance.
\end{abstract}

(C) 2005 Elsevier B.V. All rights reserved.

PACS: $29.40 . \mathrm{Gx} ; 29.40 . \mathrm{Wk}$

Keywords: Silicon sensors; Vertex detector; Irradiation

\section{Introduction}

At the Large Hadron Collider (LHC) the proton beams will collide with a centre of mass energy of $14 \mathrm{TeV}$ producing the full spectrum of B-hadrons. The $\mathrm{LHCb}$ experiment will explore the large yield of $10^{12} b \bar{b}$ pairs per year and precisely determine the CP violating observables. The only source of CP violation within the Standard Model is the phase in the quark mixing matrix. Possible additional contributions to the observed $\mathrm{CP}$ violation originating from New Physics can be revealed by using many different B-hadron decay modes and over-constraining the Standard Model parameters of the mixing matrix.

The LHCb detector is designed as a single arm spectrometer instrumenting the forward region around the $\mathrm{pp}$ interaction. Its angular acceptance ranges from 15 to $300 \mathrm{mrad}$ benefiting from the Lorentz boost of the Bhadrons and the angular correlation of the $b \bar{b}$ pairs.

A major task of the Vertex Locator (VELO) is to reconstruct the production and the displaced decay vertices of B-hadrons. This information is provided to the second level trigger and used to select events of interest. Following

\footnotetext{
${ }^{*}$ Tel.: + 41227672048.

E-mail address: doris.eckstein@cern.ch.
}

the recent overall optimisation of the LHCb experiment [1] the VELO now plays an important role in the track reconstruction. It covers the full angular acceptance of the downstream detector.

\section{VELO requirements and mechanical design}

The design of the VELO is a consequence of the demands on the precision and robustness of the vertex reconstruction, the tracking and the triggering. As the pp interaction region will be widely spread along the beam direction $\left(\sigma_{z}=5.3 \mathrm{~cm}\right)$, a series of 21 stations are arranged perpendicular to the beam over $1 \mathrm{~m}$ around the nominal interaction point allowing the reconstruction of tracks with at least three hits in the whole acceptance range.

The required precision for the reconstruction of the Bdecay vertex is only achievable with a short track extrapolation distance and minimal multiple scattering. The VELO sensors are therefore placed as close as $7 \mathrm{~mm}$ to the beam separated from it only by an aluminum RF foil. This foil, which has a maximum thickness of $300 \mu \mathrm{m}$, protects the sensor from RF pickup, the LHC vacuum from possible outgassing of the detector modules and acts as a wakefield guide. 


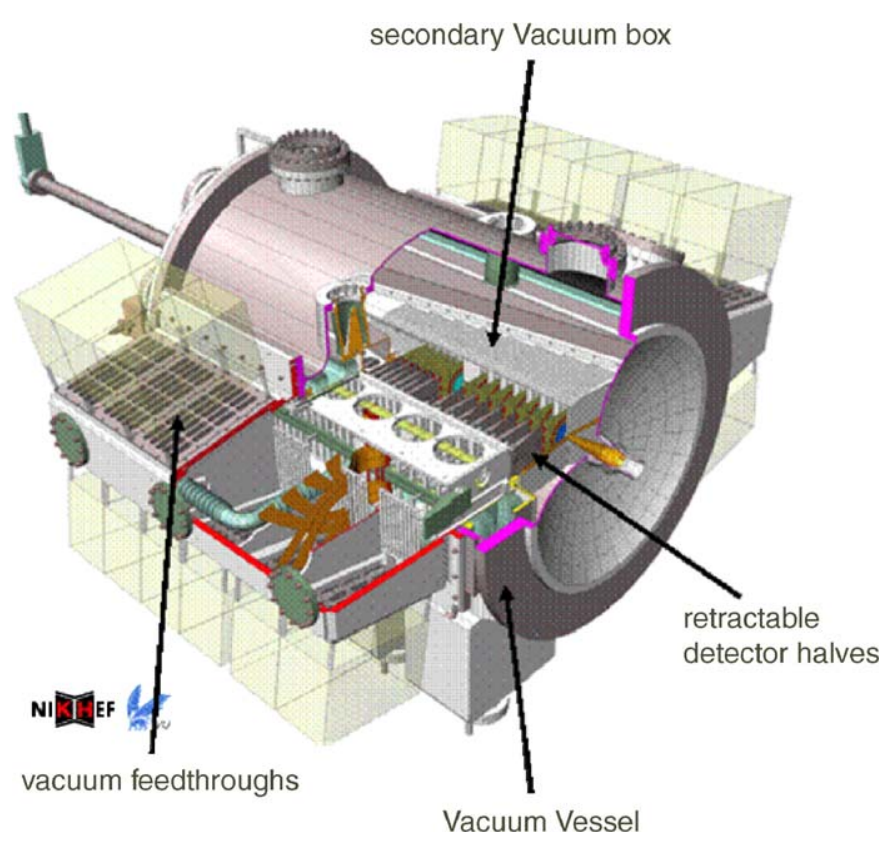

Fig. 1. Schematic of the VELO design. The main components are labelled.

Fig. 1 shows a schematic of the VELO design. The modules are placed in a vacuum box separating the beam vacuum from the detector vacuum. Another important feature is that the detector is split into two retractable halves. Thus, during the injection and acceleration phases of the $\mathrm{LHC}$ when a $3 \mathrm{~cm}$ clearance to the beam is required, the vacuum box containing the sensors will be retracted.

\section{Sensor design}

\subsection{Radiation environment}

Due to their proximity to the interaction the VELO sensors will be exposed to extreme radiation. The level of irradiation will vary across the sensor and with distance from the interaction, i.e. from station to station with levels between $5 \times 10^{12}$ and $1.3 \times 10^{14} \mathrm{n}_{\mathrm{eq}} / \mathrm{cm}^{2} /$ year for the most highly irradiated station. Due to this inhomogeneous irradiation the inverted regions in the sensor will gradually move from small to large radii and the depletion voltages will vary across the sensor. Different types of prototype sensors were studied to evaluate the best possible choice for the VELO. The results lead to the choice of oxygenated non-n sensors [2].

Due to radiation damage it is planned to replace the sensors after three years operation. Possible options for the upgraded VELO include Magnetic Czochralski [3], 3d sensors [4] and n-in-p sensors [5].

\subsection{Strip layout}

Each VELO module consists of two AC-coupled, singlesided silicon sensors mounted back-to-back: one measuring the R-and one the $\Phi$-coordinate of hits. The geometry is

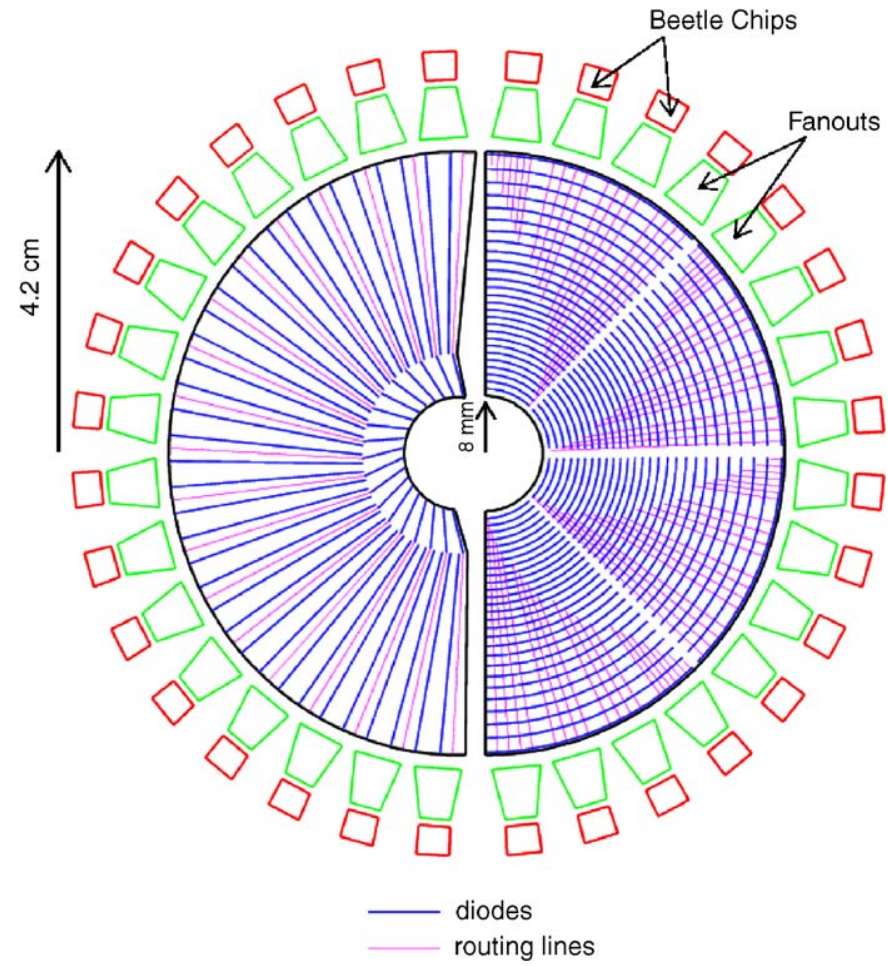

Fig. 2. Schematic of the VELO sensor design. Left: the $\Phi$-measuring sensor. Right: the R-measuring sensor.

shown in Fig. 2. It was chosen to allow for a fast standalone tracking and vertexing for the second-level trigger. Resolution and occupancy were optimised by choosing a small pitch at inner and a larger pitch at outer radii. The active area of both sensor types ranges from a radius of 8.2 to $42 \mathrm{~mm}$.

The strips of the $\Phi$-sensor are quasi-radial with a stereo angle. The sensors will be mounted flipped from station to station. In order to equalise occupancy they are split into an inner and an outer part. For the front-end chips to be placed outside of the acceptance the signals are routed to the outer part of the sensor using routing lines in a second metal layer. The pitch ranges from 36 to $97 \mu \mathrm{m}$.

The strips of the R-measuring sensor are of circular shape segmented into four azimuthal sections of $45^{\circ}$. Also here the signal is routed to the chips at the outer detector edge through a second metal layer. The smallest pitch is 40 and the largest $102 \mu \mathrm{m}$.

\section{Sensor and front-end chip performance}

\section{1. $S / N$ and overspill requirements}

The demands from the performance of the second- level trigger on the VELO have been previously reported [6]. An efficiency of $99 \%$ is required for optimal operation along with a signal-to-noise $(S / N)$ large enough to not degrade the performance by fake clusters. In order to ensure effective triggering even after irradiation the VELO aims at an initial value of $S / N>14$. 
Signal overspill can be an important contribution to trigger inefficiencies. Overspill is the signal remaining from the previous event due to the tail in the pulse shape. In case of positive overspill aligned hits will create a false track with a large impact parameter and thus fake a signal and degrade the trigger efficiency. It was shown [7] that for an overspill below $30 \%$ the trigger efficiency remains constant.

\subsection{Test beam studies}

Various prototypes of sensors and front-end chips were tested in order to find a combination suitable for the VELO and improve their designs. The first tests with a $300 \mu \mathrm{m}$ thick prototype sensor and the Beetle1.1 chip showed a $S / N \sim 18$ with a nonoptimal $36 \%$ overspill [9].

Subsequently further prototype modules were tested in the CERN SPS X7 test beam facility providing muons and pions with $120 \mathrm{GeV}$ energy in 2003 and 2004. The setup used is shown in Fig. 3. A telescope consisting of first VELO prototype sensors in R- $\Phi$-geometry can reconstruct tracks and predict clusters in the test sensor.

As the beam particles arrive asynchronous to the Beetle system clock their pulses are randomly sampled within a $25 \mathrm{~ns}$ time window. The time from a scintillator trigger to the system clock is recorded in a TDC. Reading out several consecutive Beetle pipeline locations allows the reconstruction of the whole pulse-shape.

\subsubsection{The front-end chip}

The Beetle chip [8] was chosen as front-end chip for the VELO. It is built in $0.25 \mu \mathrm{m}$ CMOS technology and is a successor of the HELIX chip developed for HERA-B. The chip integrates 128 channels with low-noise chargesensitive preamplifiers and shapers. The output is sampled with $40 \mathrm{MHz}$ into an analogue pipeline with a latency of maximum 160 sampling intervals. For analogue readout, as it will be used in the VELO and the LHCb Silicon Tracker, the data are multiplexed with $40 \mathrm{MHz}$ onto 4 ports.

The chip is a highly configurable device with several bias settings which influence pulse shape parameters such as

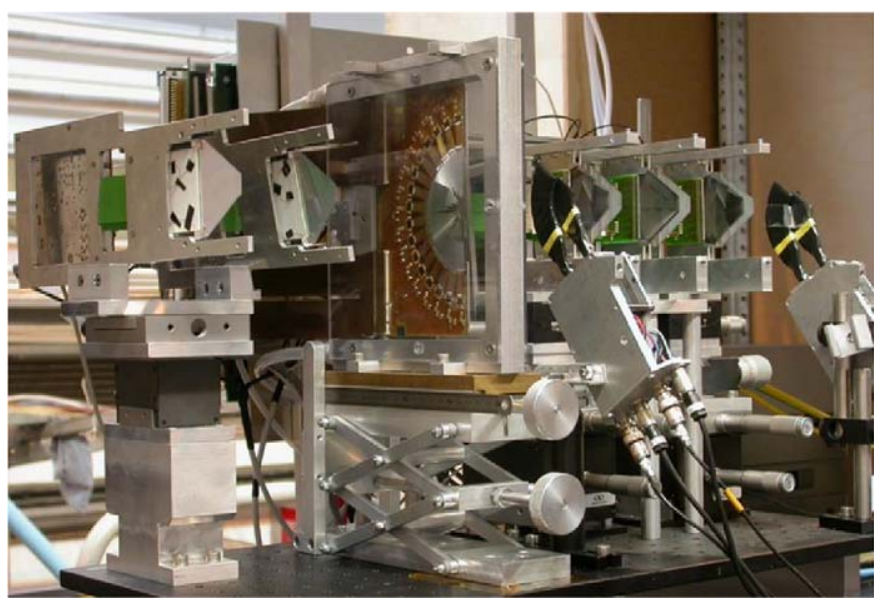

Fig. 3. Photo of the test beam setup with telescope and test module. rise-time, peak value and overspill. The most important parameters in this respect are the shaper current $\left(I_{\text {sha }}\right)$, the preamplifier current $\left(I_{\text {pre }}\right)$ and the shaper feedback voltage $\left(V_{\mathrm{fs}}\right)$.

\subsubsection{Test of a $200 \mu m$ thick sensor}

A $200 \mu \mathrm{m}$ thick n-on-n R-measuring sensor was read out through a Beetle1.2 chip mounted on a test-PCB in Summer 2003. The aim was to understand if such a thin sensor would meet the $\mathrm{LHCb}$ requirements.

The signal is calculated by summing the charge deposited in the four strips surrounding the extrapolation point of the track in the sensor. The $S / N$ is calculated as the ratio between this signal and the average noise of the strips involved. To attain the pulse shape distribution these $S / N$ values are measured as a function of TDC time. A Landau convoluted with a Gaussian is fitted and the most probable value (MPV) calculated. The signal and $S / N$ values quoted for a certain sensor refer to the peak of the pulse-shape distribution.

A scan of the main Beetle bias parameters was performed. The shaper feedback voltage appears to be the parameter influencing $S / N$ and overspill the most. It controls the resistance in the RC feedback path of the shaper amplifier. An increase in $V_{\mathrm{fs}}$ leads to a higher resistance translating into a longer rise-time, a higher signal peak and a larger overspill.

Fig. 4 shows the measurement results for the scan of this Beetle bias. As can be seen from the peak value of the pulse the maximum $S / N$ measured is 13.2. The overspill for the same setting is $45 \%$. Neither of the two values is in agreement with the $\mathrm{LHCb}$ requirements. Scanning the other chip bias parameters no better result was achieved [10].

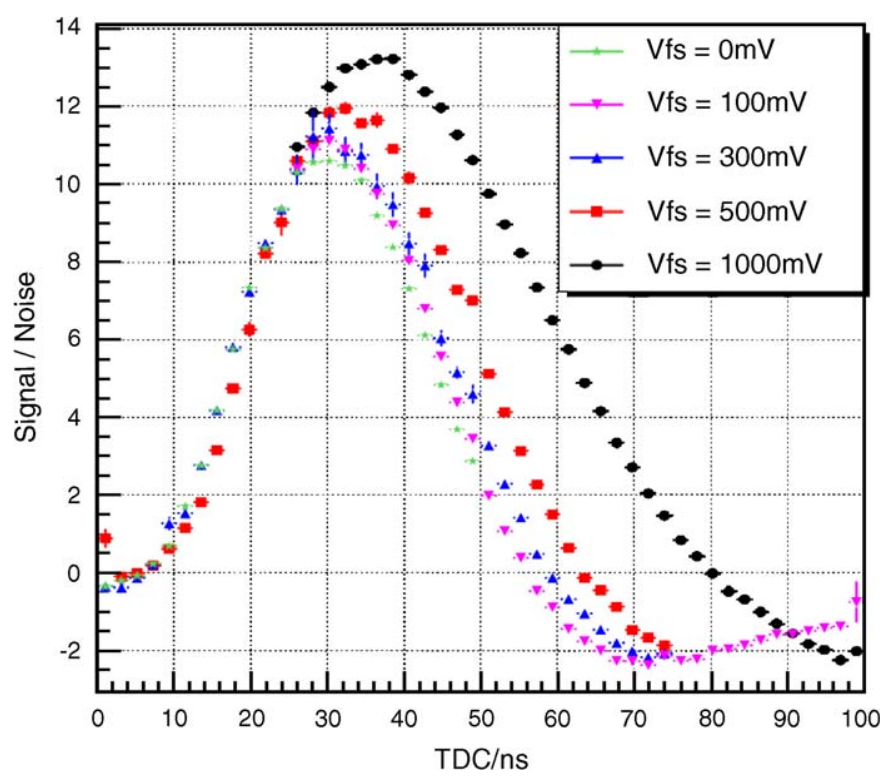

Fig. 4. Pulse shapes for different shaper feedback voltages of the Beetle1.2 reading out a $200 \mu \mathrm{m}$ thick prototype sensor. 


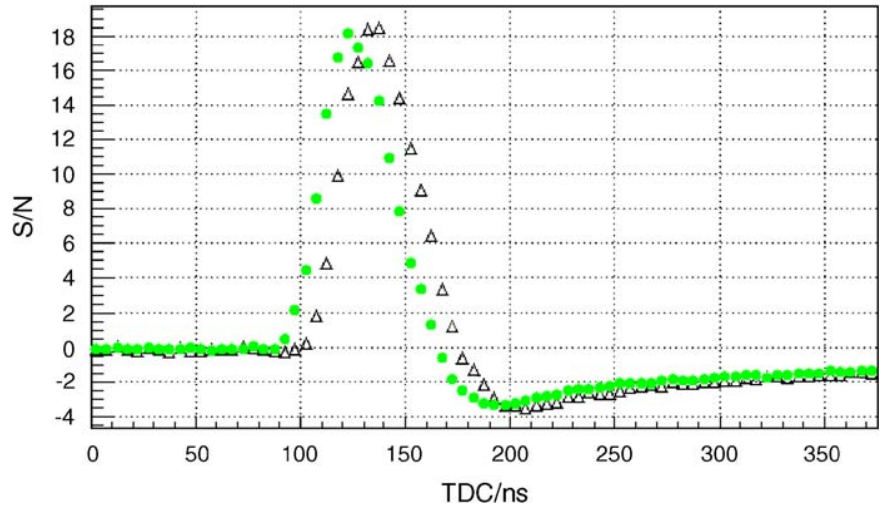

Fig. 5. Pulse shape with short (open triangles) and long (green circles) cable.

\subsubsection{Test of a $300 \mu \mathrm{m}$ thick sensor}

In spring 2004 a $300 \mu \mathrm{m}$ thick sensor of the same type was tested. The objectives were to test if an increase in sensor thickness, the use of a realistic hybrid and a more detailed investigation of the chip bias parameters would result in $S / N$ and overspill values satisfying the $\mathrm{LHCb}$ requirements.

The prototype sensor was connected to a fully populated hybrid equipped with Beetle1.3 chips. All chips were powered while one was read out. Scans of the same chip bias parameters as for the previous test were performed. The Beetle was read out in multiple time sample mode with 15 adjacent pipeline locations read out for each trigger.

For this analysis [11], no track information was considered, rather the position of the highest $S / N$ cluster in the sensor was used as a seed for the signal summation. For each of the 15 time samples a signal was measured thus allowing the reconstruction of the entire pulse shape as shown in Fig. 5.

The study of the pulse dependence on $V_{\mathrm{fs}}$ revealed that a high value of $S / N$ will be accompanied by a large overspill. Also no other Beetle bias parameter studied was able to increase the signal while decreasing the overspill. There is a range of values below $V_{\mathrm{fs}}=600 \mathrm{mV}$ within which this $300 \mu \mathrm{m}$ thick sensor satisfies the $\mathrm{LHCb}$ requirements. The ratio between the $S / N$ measurements for the two sensor types is equal to the ratio of their thickness.

\subsubsection{Analogue transmission}

The results described above were obtained with only a part of the analogue chain, i.e. short Kapton connection from the hybrid to the repeater board and $15 \mathrm{~m}$ cat 6 cables further to the ADC boards. In the $\mathrm{LHCb}$ experiment the ADCs will be placed in an accessible and radiation free area. Thus the signals will be routed through a $60 \mathrm{~m}$ cat 6 cable which might introduce pulse distortions which will be seen as cross talk to the next channel in the data. In order to correct for this an equalisation in form of a precompensation is introduced.

Fig. 5 shows the measured pulse shapes for the short $15 \mathrm{~m}$ cable without equalisation compared to the pulse after the $60 \mathrm{~m}$ cable with equalisation. No significant difference in $S / N$ and overspill were observed.

\section{Summary}

Beam test results from prototype sensors of $200 \mu \mathrm{m}$ and $300 \mu \mathrm{m}$ thickness show that the $S / N$ values scale in proportion with the thickness. The measured values of $S / N$ and overspill for the $300 \mu \mathrm{m}$ thick prototype sensor satisfy the $\mathrm{LHCb}$ requirement.

\section{References}

[1] The LHCb Collaboration, Status of the Detector Reoptimisation, CERN/LHCC/2003-003, 2003.

[2] The LHCb Collaboration, LHCb Vertex Locator Technical Design Report, CERN/LHCC/2001-011, 2001.

[3] A. Bates, et al., Results from the first test beam of a large Microstrip Czochralski Silicon Detector equipped with LHC speed electronics, LHCb-2004-052, 2004.

[4] RD50 Status Report 2004, CERN/LHCC/2004-031, 2004.

[5] G. Casse, et al., Nucl. Instr. and Meth. A 535/1-2 (2004) 362.

[6] P. Koppenburg, Simulation of the vertex trigger preprocessor: Effect of noise on L1 performance, LHCb 99-003, 1999.

[7] P. Koppenburg, Effect of Pulse Overspill on the Level 1 Trigger, LHCb 2001-078, 2001.

[8] N. van Bakel, et al., The Beetle Reference Manual, in preparation.

[9] S. Klous, et al., Characteristics of 16 Beetle1.1 chips on a VELO hybrid, LHCb 2003-069, 2003.

[10] J. Buytaert, et al., Performance of a Beetle1.2 chip reading out a Micron PR03 R measuring sensor, LHCb 2003-135, 2003.

[11] J. Palacios, et al., Pulseshape characteristics of a $300 \mu \mathrm{m}$ PR03 Rmeasuring VELO sensor read out with a Beetle 1.3 chip, LHCb 2004 068 VELO, 2004. 